\title{
Urinary mycobacterium avium presenting as sterile pyuria
}

\author{
Kai Yang,; Mary Samplaski, MD, ${ }^{2}$ Tony Mazzulli, MD, Kirk Lo, MD, Ethan Grober, MD, ${ }^{4}$ Keith Allen Jarvi, $M D^{4}$ \\ 'Faculty of Medicine, University of Toronto, Toronto, ON, Canada; 2 Department of Urology, University of Southern California, CA, U.S.; 3 Department of Laboratory Medicine and Pathobiology, University of \\ Toronto, Toronto, ON, Canada; ${ }^{4}$ Department of Surgery, University of Toronto, Toronto, ON, Canada
}

Cite as: Can Urol Assoc J 2016;10(5-6):E186-8. http://dx.doi.org/10.5489/cuaj.3363 Published online May 12, 2016.

\section{Abstract}

A 65-year-old healthy woman presented with persistent, asymptomatic sterile pyuria detected by her family physician. While she did not have symptoms, the patient recounts that she has had cloudy urine for years. Cultures of the urine for bacteria showed no growth and no fungi were identified. First-morning urine samples were sent for both tuberculosis and nontuberculosis mycobacterium species testing. The culture grew genotypically identified Mycobaterium avium complex (MAC). Mantoux skin testing was positive. No urological abnormalities were detected by ultrasound and computed tomography (CT) imaging of the urinary tract.

\section{Introduction}

MAC is classified as a slow-growing nontuberculous mycobacterium (NTM). ${ }^{1}$ While MAC is ubiquitous in the environment and is the most common disease-causing NTM in humans, there are very few reported cases of MAC infection in the urine and only two reported cases of asymptomatic MAC urine infection. Predisposing factors for NTM infections include defects in host immunity and direct exposure to the NTMs. MAC is ubiquitous in the soil, so those working with soil may be at higher risk of MAC infections.

The diagnosis of a MAC urine infection is made with urine culture for MAC and/or polymerase chain reaction (PCR). Direct staining for acid-fast bacilli is considered unreliable. For most patients with a MAC infection, skin Mantoux testing is positive.

Testing for MAC infection should be considered for patients with sterile pyuria. While MAC urine infections are not commonly reported, this may reflect how commonly testing for MAC infection is performed rather than how commonly MAC infection occurs.

\section{Case report}

A 65-year-old woman presented to general urology clinic with persistent, asymptomatic sterile pyuria. This was detected incidentally on routine urinalysis performed by her primary care physician. However, on further questioning, she reported having had cloudy urine for years. Several urinalyses performed by her primary care physician demonstrated abundant neutrophils, but cultures for bacteria and fungus showed no growth and microscopic examination for parasites was negative. She denied any history of dysuria, hematuria, fevers, chills, or other systemic complaints.

The patient had a relatively unremarkable medical history with the only medical issues being psoriasis, hypertension, and hypercholesterolemia. She was born in the Czech Republic and emmigrated to Canada at 20 years of age. There was no family history of tuberculosis. She had no antecedent travel or exposure to sick individuals.

The patient's physical examination was unremarkable and her repeated urinalyses showed 5-10 leukocytes per high-powered field on urine microscopy. This urine sample again demonstrated no growth on routine culture. Due to the patient's history of repeated sterile pyuria, a first-morning urine was sent for culture for Mycobaterium tuberculosis, Mycobaterium avium, fungus, and examination for parasites. The culture grew genotypically identified Mycobaterium avium complex (MAC).

Mantoux skin test was performed and read as positive with $17 \mathrm{~mm}$ of induration. Ultrasound and computed tomography (CT) scan of the abdomen and pelvis demonstrated no abnormalities. Consideration was given to medical treatment of the MAC, but given that the patient was completely asymptomatic, the consensus decision was to manage her conservatively.

\section{Discussion}

Based on its microbiology, clinical, and epidemiologic characteristics, Mycobacterium avium is classified as a slow-growing nontuberculous mycobacterium (NTM). ${ }^{1}$ It is 
one of the most common nontuberculous species causing human disease in the U.S. ${ }^{2}$ The precise frequency of disease due to Mycobacterium avium is unknown because disease reporting is not mandatory.

As a nontuberculous mycobacterium, Mycobacterium avium is ubiquitous in the environment and it has been recovered from water and soil, as well as domestic and wild animals. ${ }^{3-5}$ Environmentally and clinically isolated MACs generally belong to different serotypes. ${ }^{6}$ However, mycobacteria can become aerosolized from aqueous sources, and the strains that are more easily aerosolized are phenotypically the same as the ones that cause pulmonary infections. ${ }^{7,8}$ Isolates similar or identical to clinically isolated strains have also been recovered from naturally occurring surface water, hot tubs, and piped hot water systems. Currently, there are no convincing data demonstrating human-to-human transmission. Thus, the concept prevails that these organisms are acquired from the environment. ${ }^{5-8}$

Risk factors for nontuberculous mycobacterial disease include defects in the host immune response due to inherited or acquired conditions, particularly those that affect the Th1 cell and macrophage pathways, and exposure to environmental NTMs; repeated surgical interventions in the genitourinary tract could also introduce environmental MAC..$^{9}$ There was no evidence that our patient had any recognized risk factors for MAC infection, nor any signs of being immune-compromised.

Unlike genitourinary tuberculosis, genitourinary infections caused by MAC are relatively rare and only a few cases have been reported..$^{10-12}$ To date, published reports of MAC infection of the genitourinary tract system have only reported its detection in urine samples, while other NTMs have been detected in prostate and epididymis biopsy samples. In nearly all reported cases, patients have symptoms of infection similar to other conventional bacterial genitourinary infections, including fever, dysuria, hematuria, urgency, and urinary frequency. ${ }^{10-12}$ There have been only two reported cases of asymptomatic infection. ${ }^{10,11}$

Detection of MAC through urine culture and/or polymerase chain reaction (PCR) is most commonly used to establish the diagnosis, though direct staining for acid-fast bacilli has also been used, but is thought to be unreliable. ${ }^{10,11}$ Most reported MAC-infected patients had a positive Mantoux test and persistent pyuria. In some studies, histological study was performed on prostate tissue and caseating granulomatous lesions were found..$^{10,12}$ However, due to the invasiveness of tissue biopsy, this investigation is not regularly performed in other studies. Other radiological urinary tract infection findings, such as hydronephrosis and multiloculated renal cysts, were only reported by Hung et al. ${ }^{11}$

The etiology of MAC infection in the genitourinary tract system is poorly understood. Potential sources of MAC infection include introduction of the organism through surgical manipulation of the urinary tract system, trauma with subsequent contamination, ${ }^{10}$ or hematogenous or lymphatic spread from other organs, such as lungs. ${ }^{11}$ Our patient had no signs/symptoms suggesting infection of other organ systems or any of the other risk factors.

The treatment for genitourinary MAC infection is not well-defined and the reported cases have been treated on an individualized basis. Treatments reported in previous reports have included expectant management, medical treatment, and surgical treatment. Medical management of mycobacterium infection, such as isoniazid, streptomycin, rifampin, and ethambutol, are oftentimes ineffective against MAC infection, but may be used depending on culture and in vitro susceptibility results. ${ }^{10,12}$ Due to the asymptomatic nature of our patient, as well as her normal imaging, she was treated with conservative management.

Genitourinary tract infections due to Mycobacteria tuberculosis are comparatively more common than NTM infections. An estimated $4-20 \%$ of patients with pulmonary tuberculosis develop genitourinary tract involvement. ${ }^{13}$ The clinical presentation of genitourinary tuberculosis infection and NTM infection generally both manifest with lower urinary tract symptoms. Based on Huang et al, patients with NTM infection are more likely to report constitutional symptoms, such as fever and leukocytosis, and shorter duration of the symptoms (one month or less) than those with tuberculosis infections, which typically last up to three months. ${ }^{11}$

This case study is one of the only reports of MAC infection in the urine of an immune-competent individual. Despite the report of other NTM species in genitourinary organs, such as prostate and epididymis, detection of MAC was only reported in urine samples. Although rare, genitourinary MAC infections should be considered in the differential diagnosis of sterile pyuria, especially in patients who are resistant to conventional antibiotic treatment. At present, treatment is generally individualized and may include surveillance, as with our patient.

Competing interests: Dr. Mazzulli has been an Advisory Board member for Merck, Paladin, and Roche; a Speakers' Bureau member for Merck and Paladin; and has participated in clinical trials for Qvella. Dr. Grober has been an Advisory Board and Speakers' Bureau member for Eli Lilly, Merck, and Paladin; has received grants/honoraria from Paladin; and holds investments in MHB Labs. Dr. Jarvi has been an Advisory Board member for Eli Lilly; has received grants/honoraria from Allergan; and has participated in clinical trials for Allergan. The remaining authors declare no competing financial or personal interests.

This paper has been peer-reviewed. 
Yang et al.

\section{References}

1. Runyon EH. Anonymous mycobacteria in pulmonary disease. Med Clin North Am 1959;43:273-90.

2. Good RC, Snider DE Jr. Isolation of nontuberculous mycobacteria in the U.S., 1980. J Infect Dis 1982;146:829-33. http://dx.doi.org/10.1093/infdis/146.6.829

3. Wolinsky E, Rynearson TK. Mycobacteria in soil and their relation to disease-associated strains. Am Rev Respir Dis 1968;97:1032-7.

4. Goslee S, Wolinsky E. Water as a source of potentially pathogenic mycobacteria. Am Rev Respir Dis 1976;113:287-92.

5. Gruft H, Falkinham J0 3rd, Parker BC. Recent experience in the epidemiology of disease caused by atypical mycobacteria. Rev Infect Dis 1981;3:990-6. http://dx.doi.org/10.1093/clinids/3.5.990

6. Horsburgh RC Jr. Epidemiology of Mycobacterium avium complex. In: Mycobacterium Avium Complex Infection, Korvick JA, Benson CA (Eds), Marcel Dekker, Inc, New York 1996. p.1.

7. Meissner $G, A n z W$. Sources of Mycobacterium avium complex infection resulting in human diseases. Am Rev Respir Dis 1977:116:1057-64.

8. Meissner PS, Falkinham J0 3rd. Plasmid DNA profiles as epidemiological markers for clinical and environmental isolates of Mycobacterium avium, Mycobacterium intracellulare, and Mycobacterium scrofulaceum. J Infect Dis 1986;153:325-31. http://dx.doi.org/10.1093/infdis/153.2.325
9. Al-Muhsen S, Casanova JL. The genetic heterogeneity of mendelian susceptibility to mycobacterial diseases. J Allergy Clin Immunol 2008;122:1043-51. http://dx.doi.org/10.1016/i.jaci.2008.10.037

10. Cark R, Cardona L, Valainis $G$, et al. Genitourinary infections caused by mycobacteria other than Mycobacterium tuberculosis. Tubercle 1989;70:297-300. http://dx.doi.org/10.1016/00413879(89)90025-1

11. Huang $\mathrm{CT}$, Chen $\mathrm{CY}$, Chen HY, et al. Genitourinary infections caused by nontuberculous mycobacteria at a university hospital in Taiwan, 1996-2008. Clin Microbiol Infect 2010;10:1585-90. http://dx.doi. org/10.1111/i.1469-0691.2010.03180.x

12. Mikolich DJ, Mates SM. Granulomatous prostatitis due to Mycobacterium avium complex. Clin Infect Dis 1992;14:589-91. http://dx.doi.org/10.1093/clinids/14.2.589

13. Abbara A, Davidson RN. Etiology and management of genitourinary tuberculosis. Nat Rev Urol 201 1;8:67888. http://dx.doi.org/10.1038/nrurol.2011.172

Correspondence: Dr. Keith Allen Jarvi, Department of Surgery, University of Toronto, Toronto, ON, Canada; kjarvi@mtsinai.on.co 